\title{
Larval Polytene Chromosomes of Five Species of Blackflies (Diptera: Simuliidae) from Doi Inthanon National Park, Northern Thailand
}

\author{
Chaliow Kuvangkadilok ${ }^{1, *}$, Suwannee Phayuhasena ${ }^{1}$ \\ and Chainarong Boonkemtong ${ }^{2}$ \\ ${ }^{1}$ Department of Biology, Faculty of Science, Mahidol University, Rama VI Road, Bangkok 10400, Thailand \\ ${ }^{2}$ National Center for Genetic Engineering and Biotechnology, National Science and Technology Development Agency, \\ Rama VI Road, Bangkok 10400, Thailand \\ Accepted March 3, 1999
}

\begin{abstract}
Summary The standard photographic maps of five blackfly species from northern Thailand: i.e., Simulium (Nevermannia) caudisclerum, S. (Simulium) fenestratum, S. (S.) nakhonense, S. (S.) rufibasis and $S$. (Montisimulium) sp. G were constructed from larval salivary gland polytene chromosomes and are described herein. All the five species have three pairs of chromosomes $(2 n=6)$ which are arranged from the longest to the shortest. Chromosome I of all species are metacentric while most of the chromosome II and III are submetacentric. The centromeric regions of all chromosomes of $S$. (S.) nakhonense and $S$. (S.) rufibasis were expanded whereas the heavy centromeric bands were presented in $S$. (N.) caudisclerum, $S$. (S.) fenestratum and $S$. (M.) sp. G. The nucleolar organizer of $S$. (N.) caudisclerum, $S$. (S.) fenestratum, $S$. (S.) nakhonense and $S$. (M.) sp. G is situated near the centromeric band of chromosome I. On the other hand, $S$. $(S$.) rufibasis has the nucleolar organizer on chromosome II. The Balbiani ring and double bubble are located near the tip of short arm of chromosome III in all species except for $S$. (S.) fenestratum, in which they are detected on chromosome II. Moreover, two types of B chromosomes and pseudochromocenters were also found in some larvae of $S$. (S.) nakhonense. There are no inversion polymorphisms detected in wild populations of these species. The five Simulium species have specific and fixed standard banding sequences which are different among species, although some banding sequences in chromosome arm IIIS show homology.
\end{abstract}

Simuliidae, or blackflies, a small family of Diptera, consists of approximately 24 genera and 1571 described species (Crosskey 1993). Blackflies has been studied at the morphotaxonomic level in various parts of the world (e.g., Crosskey 1960, Datta 1974a, b, Davies 1968, Takaoka 1977, 1979, Takaoka and Davies 1995, Takaoka and Adler 1997). The most of blackflies are recognized and defined on external morphological characters of larvae, pupae and adults. However, blackflies are more difficult to classify than most other insect groups because their morphologies are similar among species (Crosskey 1981) as well as sibling species (Procunier and Muro 1993).

Larval polytene chromosomes of blackflies (Simuliidae) as well as in other families and genera of the Diptera, e.g. the Drosophilidae, Anophelines, Chironomidae and Tephritidae (MavraganiTsipidou et al. 1994, Sukowati and Baimai 1996, Wülker et al. 1989, Procunier and Smith 1993) have been proved to be an excellent material not only in the studies of cytotaxonomy (Vajime and Dunbar 1975, Rothfels 1979, Dunbar and Vajime 1981, Procunier and Muro 1993), chromosomal polymorphisms (Bedo 1979, Rothfels and Freeman 1977), phylogenetic relationships (Bedo 1979, Rothfels 1981), but also to identify members of sibling species complexes (Bedo 1977, Conn et al. 1989, Boakye 1993, Hadi et al. 1996b).

Knowledge of cytotaxonomy of blackflies from Thailand is very scarce, only one report on

\footnotetext{
* Corresponding author.
} 


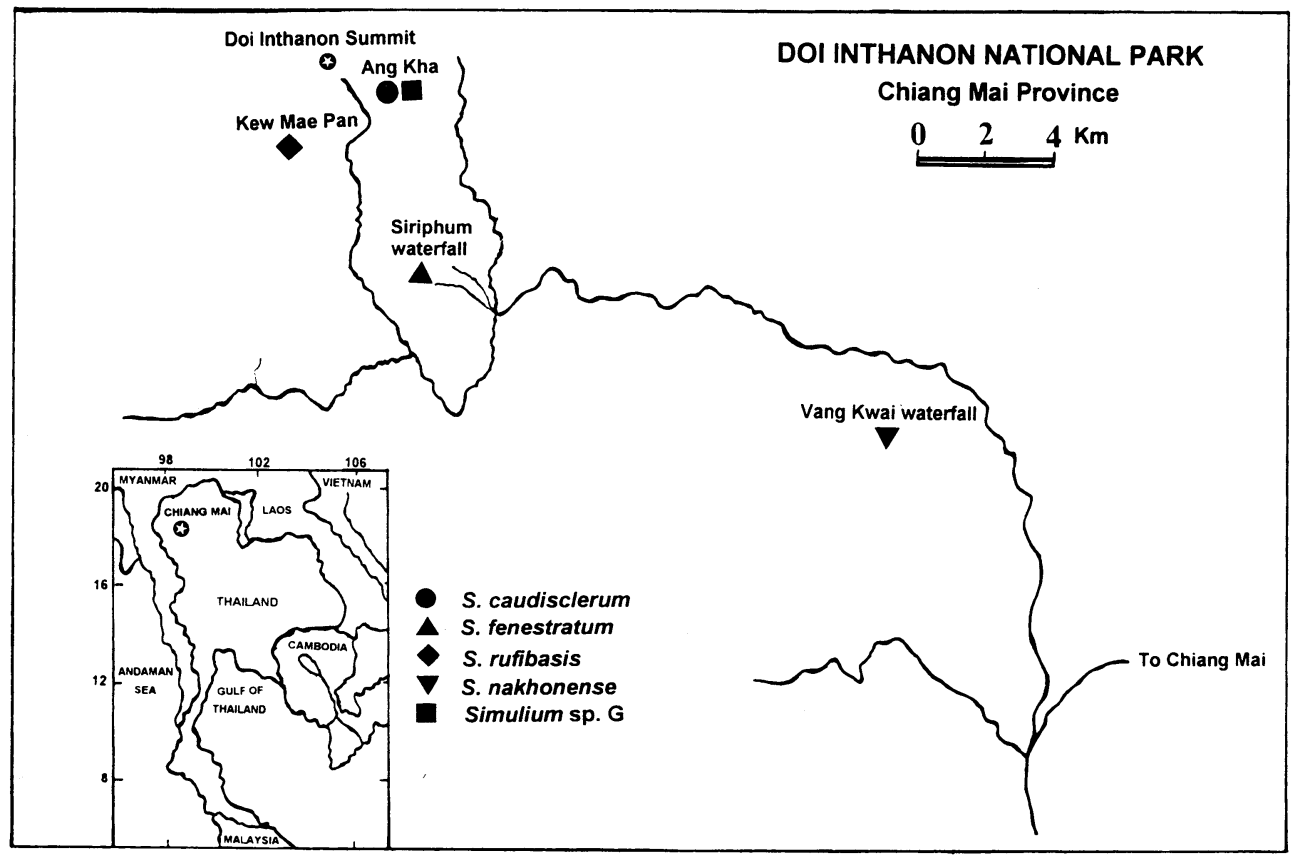

Fig. 1. Map of Doi Inthanon National Park, Chiang Mai Province, showing the 4 locations where the 5 Simulium species were collected for this study.

Table 1. List of five Simulium species from different localities of Doi Inthanon National Park used in this study

\begin{tabular}{lll}
\hline \multicolumn{1}{c}{ Species } & \multicolumn{1}{c}{ Localities (altitude) } & Date of Collection \\
\hline$S .(N$.$) caudisclerum$ & Ang Kha $(2460 \mathrm{~m})$ & August 1997 \\
S. $($ S. $)$ fenestratum & Siriphum waterfall $(1270 \mathrm{~m})$ & August 1997 \\
S. $(S$.$) nakhnense$ & Vang Kwai waterfall $(410 \mathrm{~m})$ & October 1997 \\
$S .(S$.$) rufibasis$ & Kew Pae Pan $(2300 \mathrm{~m})$ & May 1997 \\
$S .(M$.$) sp. G$ & Ang Kha $(2460 \mathrm{~m})$ & August 1997 \\
\hline
\end{tabular}

population cytogenetic studies of Simulium feuerborni Edwards from northern Thailand has been presented (Kuvangkadilok et al. in press). Recently a total of 17 Simulium species consisting of 13 known species, 3 unnamed species and 1 new species has been recognized at Doi Inthanon National Park, northern Thailand, on the basis of external morphological characters of larvae and pupae (Kuvangkadilok, unpublished data). It is the aim of this project to investigate cytotaxonomically blackflies belonging to the subgenera Nevermannia Enderlein, Montisimulium Rubtsov and Simulium Latreille s. str. of the genus Simulium Latreille s. 1., which are distributed in various localities at Doi Inthanon National Park, northern Thailand.

In this report, we present the standard photographic maps of the larval polytene chromosomes for 5 Simulium species: i.e., S. (N.) caudisclerum, S. (S.) fenestratum, S. (S.) nakhonense, S. (S.) rufibasis and $S$. (M.) sp. G collected from different locations at Doi Inthanon National Park, northern Thailand.

Materials and methods

Larval specimens of 5 Simulium species were collected in May, August and October 1997 from four locations at different altitudes of Doi Inthanon National Park, Chiang Mai Province (Fig. 


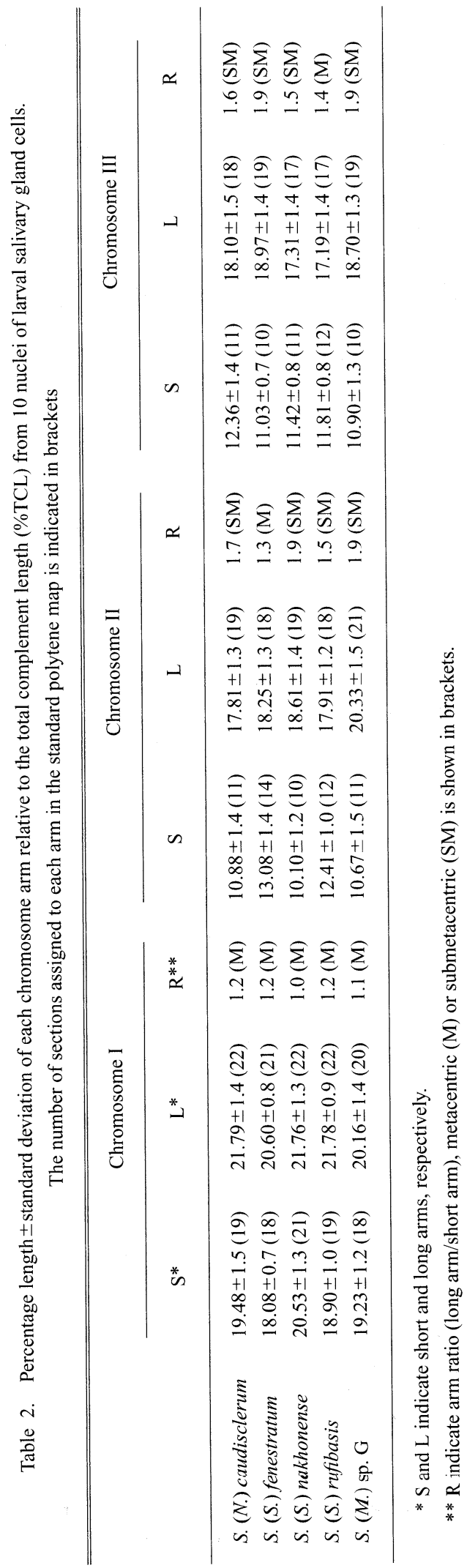


1, Table 1). Larvae were picked up by small forceps off submerged fallen leaves and trailing water glasses. They were preserved in two changes of freshly prepared Carnoy's fixative $(2: 1$, absolute ethanol: glacial acetic acid). The preserved larvae were stored in a freezer $\left(-18^{\circ} \mathrm{C}\right)$ until required for slide preparation. Larval identification was carried out following keys of Takaoka and Suzuki (1984), Takaoka and Davies (1995) and Takaoka and Saito (1996). The specimens of $S$. (N.) caudisclerum, $S$. (S.) nakhonense, $S$. (S.) fenestratum, $S$. (S.) rufibasis and $S$. (M.) sp. G a new species were kindly confirmed and identified by Professor Hiroyuki Takaoka, Department of Infectious Disease Control, Faculty of Medicine, Oita Medical University, Japan.

Only last stage or penultimate-instar larvae were used for salivary gland polytene chromosome preparations. The polytene chromosomes were stained with $1.6 \%$ Orcein (lactic acid:propionic acid: water, $2: 2: 1$ ) for 15-20 min using the methods described by Porter and Martin (1977). The well spread polytene chromosomes were photographed on Kodak technical pan film under oil immersion $(\times 330)$, with a green filter. The relative lengths of the 3 chromosome pairs and their respective arms were measured from photographs of ten well spread polytene chromosome complements. Chromosome mapping conventions and nomenclature follow the method described by Bedo (1977).

\section{Observations}

\section{General chromosome morphology}

All 5 species of Simulium have 3 pairs of chromosomes $(2 n=6)$. Each chromosome consists of 2 arms which may be equal or unequal in length depending on the position of the centromere. Table 2 shows the mean relative lengths of the polytene chromosome arms and the number of sections assigned to each arm in the standard map. The detail of polytene chromosomes of 5 species of Simuli$u m$ which are studies here are described below.

\section{S. (N.) caudisculerum}

Chromosome I is metacentric and the longest of the complement. It is $41 \% \mathrm{TCL}$, divided into 41 sections (1-41). The short arm (IS) extends from sections 1-19 while the long arm (IL) contains sections $20-41$ (Fig. 2). The short arm is characterized by the presence of a nucleolar organizer (NO) in section 19 located near a heavily stained band of centromere in section 20A. The long arm have a prominent dark band in section $20 \mathrm{~B}$ very close to the centromere and a series of five bands in section 21.

Chromosome II is submetacentric and remarkably shorter than chromosome I. It is divided into 30 sections (42-71). The short arm (IIS) has the puffy euchromatic region named the blister in section $45 \mathrm{~B}$. The long arm is differentiated by a prominent series of dark bands in sections $70 \mathrm{C}-$ $71 \mathrm{AB}$ toward the distal end. Additionally, a series of bands in sections $65 \mathrm{~B}-66 \mathrm{C}$ is also found in this long arm.

Chromosome III is also submetacentric and contains sections 72-100. This chromosome is almost equal in length with chromosome II. The short arm (IIIS) is recognized by the double bubble $(\mathrm{db})$ and the Ring of Balbiani (BR) in sections $76 \mathrm{C}$ and $77 \mathrm{C}$ respectively. Moreover, the presence of the 3 dark bands in sections $81 \mathrm{C}-82 \mathrm{AB}$ adjacent to the centromere is also a useful cytological landmark for the short arm. The long arm (IIIL) has a prominent series of bands in section 93 and two heavy bands in section 100B near the distal end of the arm.

There is no inversion found in all three chromosomes.

\section{S. (S.) fenestratum}

Chromosome I is metacentrikc with 39\% TCL. The short arm and the long arm consist of sections 1-18 and 19-39 respectively (Fig. 3). A prominent nucleolar organizer is found in section 


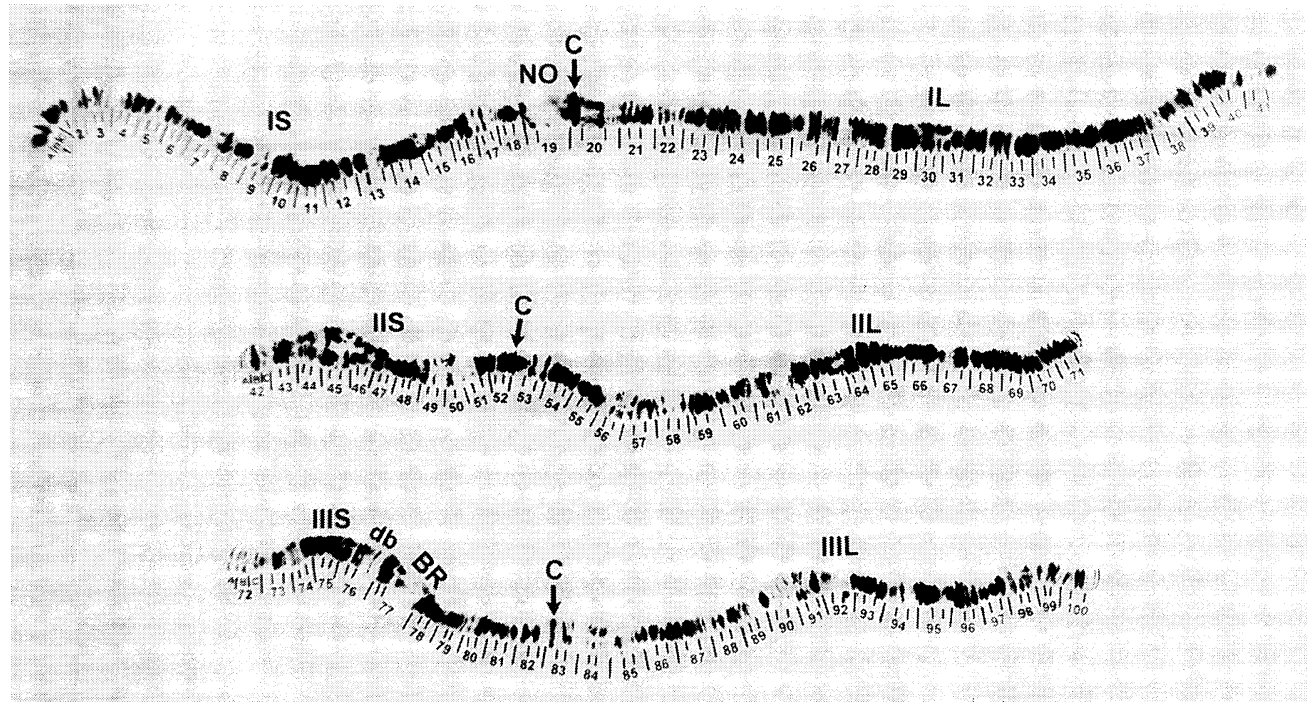

Fig. 2. Standard photographic map of salivary gland polytene chromosomes of Simulium (Nevermannia) caudisclerum. I. II. III = chromosome I, II, III, S= short arm, $\mathrm{L}=$ long arm, $\mathrm{C}=$ centromere, $\mathrm{NO}=$ nucleolar organizer, $\mathrm{BR}=$ Ring of Balbiani, $\mathrm{db}=$ double bubble.

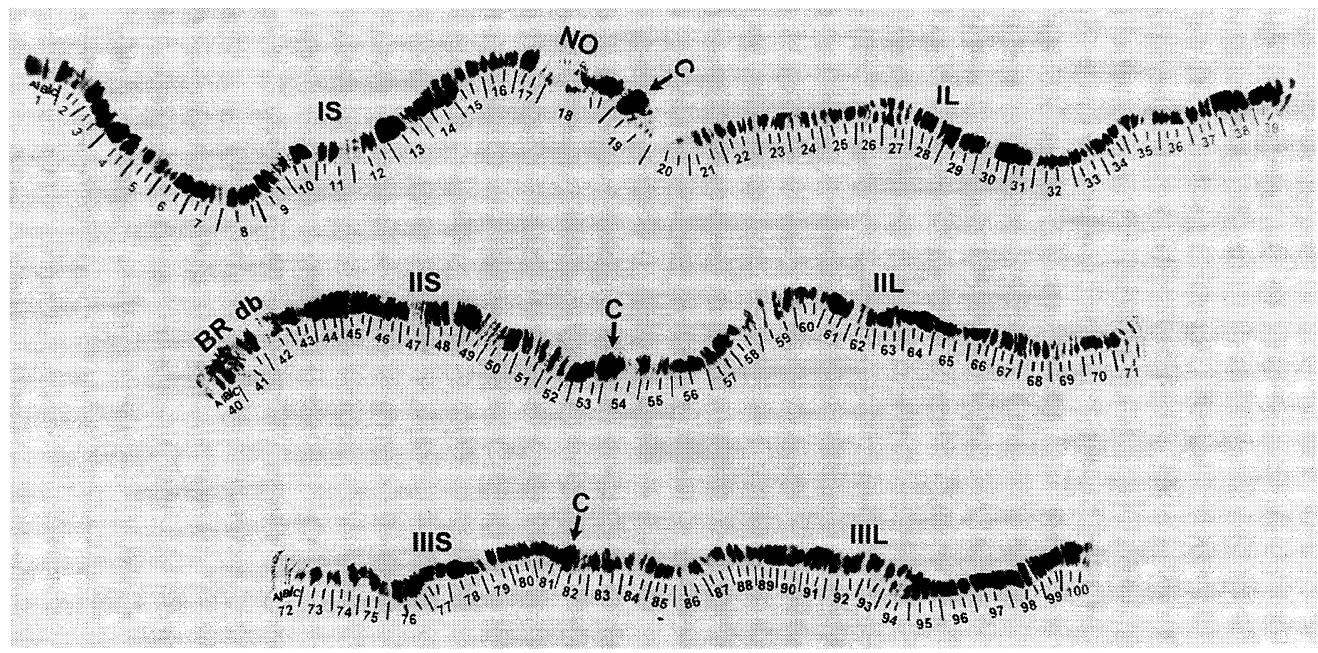

Fig. 3. Standard photographic map of salivary gland polytene chromosomes of Simulium (Simulium) fenestratum. Symbols and designations as in Fig. 2.

18A of the short arm. In addition, a group of fine bands at the tip of this arm serves as a useful marker. The long arm is recognized by the presence of a series of dark bands in sections 38-39 toward the distal end.

Chromosome II somewhat shorter than chromosome I. It is metacentric and divided into 32 sections (40-71). This chromosome is easily distinguished by the ring of Balbiani near the tip of the short arm in section 41A adjacent to the double bubble in section 42A. The long arm is characterized by flared tip.

Chromosome III, the shortest chromosome complement, is submetacentric with about $29 \%$ TCL. It is divided into 29 sections (72-100). The tip of the short arm is flared. There is a series of 


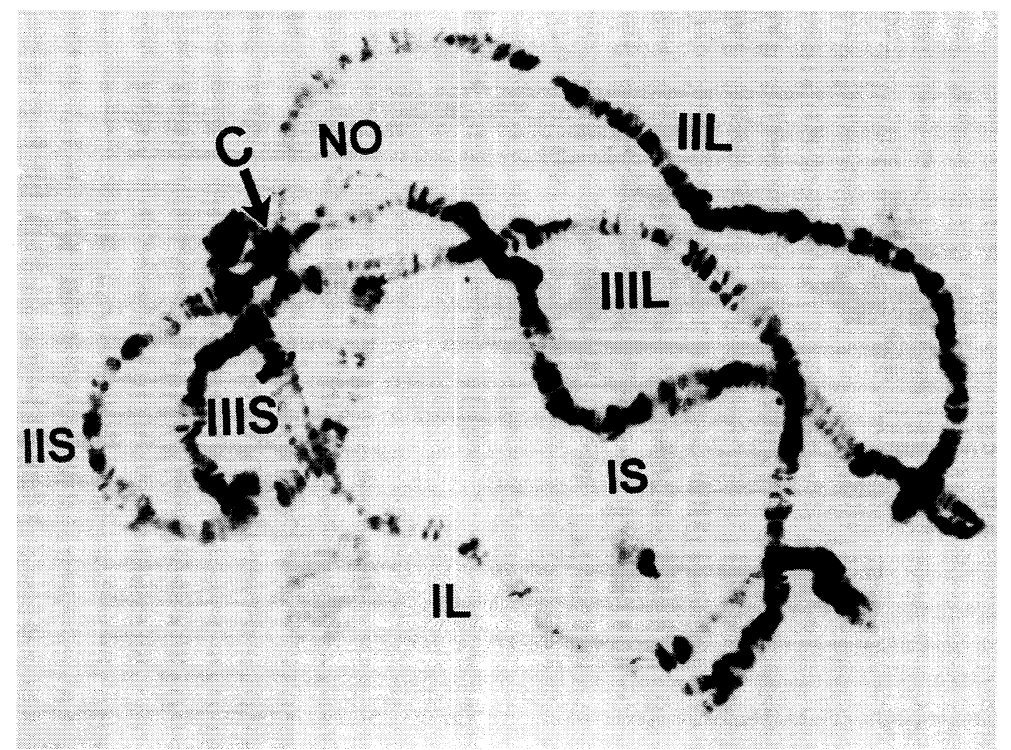

Fig. 4. Polytene chromosome complement of Simulium (Simulium) nakhonense. Symbols and designations as in Fig. 2.

dark bands in sections $99-100 \mathrm{AB}$ toward the end of long arm.

No inversions have been detected in any chromosomes.

\section{S. (S.) nakhonense}

Chromosome I, II and III of S. (S.) nakhonense collected from Vang Kwai waterfall are joined together at their centromeres (ectopic pairing) forming a loose "pseudochromocenter" in 79 larvae out of 180 examined larvae (Fig. 4). However, most of larvae collected from the same locality are nonchromocenter. Moreover, two types of supernumerary chro-

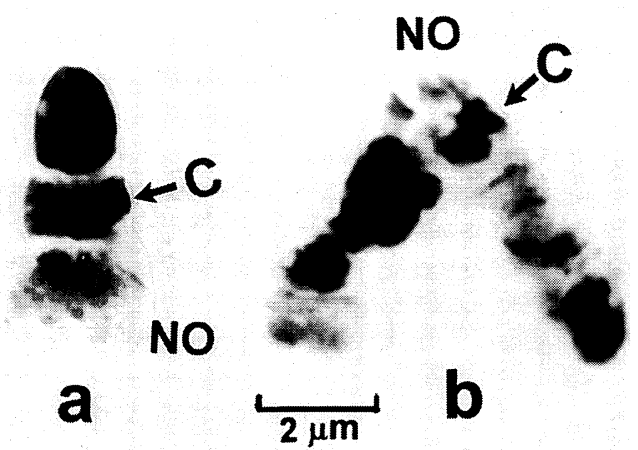

Fig. 5. The supernumerary chromosomes (B chromosomes) of Simulium (Simulium) nakhonense. $\mathrm{C}=\mathrm{cen}$ tromere, $\mathrm{NO}=$ nucleolar organizer. mosomes (B chromosomes) with heavy heterochromatin in a small metacentric shape occurred independently in 13 larvae of $S$. (S.) nakhonense (Fig. 5).

Chromosome I is easily identified by the short arm (sections 1-21) and the long arm (sections 22-43) of almost equal length (Fig. 6). The presence of a prominent nucleolar organizer is a useful marker for the short arm. A group of 4 bands in sections $38 \mathrm{AB}$ is found in the long arm.

Chromosome II is submetacentric. The higher arm ratio serves to distinguish chromosome II from Chromosome III. The short arm has a flared tip. The long arm shows a group of heavy dark bands in sections $70 \mathrm{AB}$.

Chromosome III is submetacentric. The short arm is easily recognized by the Ring of Balbiani, the double bubble and the dark bands adjacent to the centromere. A series of dark bands in section 99 is a good character for the long arm.

No inversions have been found in all three chromosomes. 


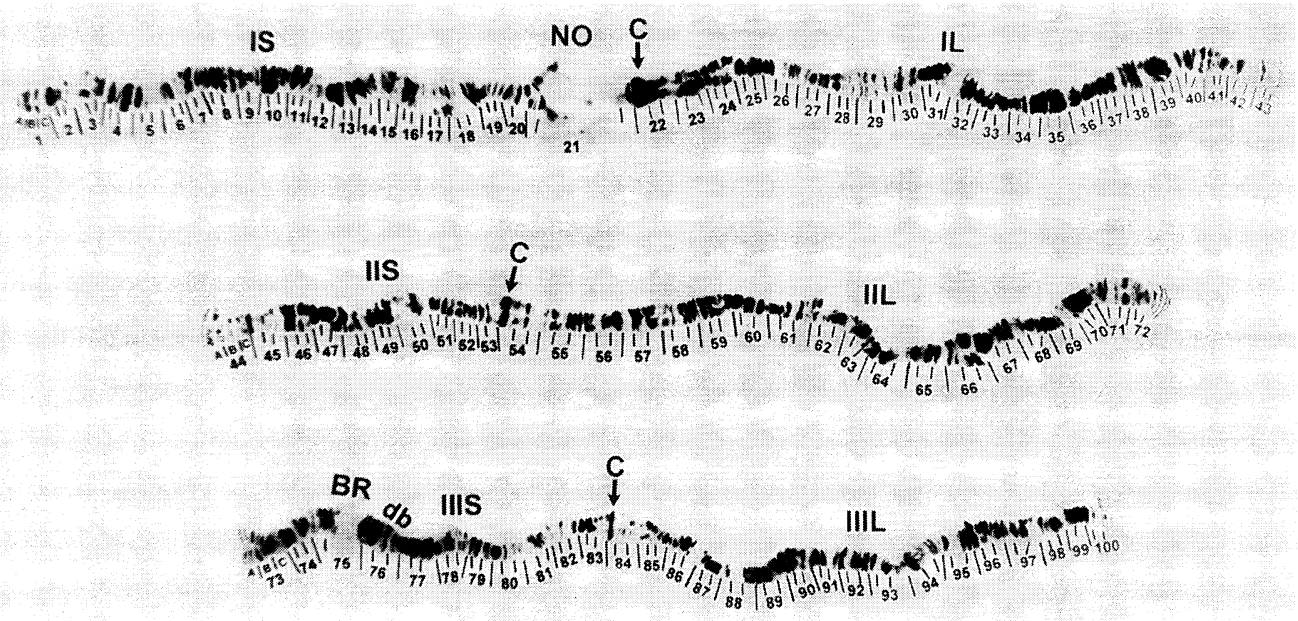

Fig. 6. Standard photographic map of salivary gland polytene chromosomes of Simulium (Simulium) nakhonense. Symbols and designations as in Fig. 2.

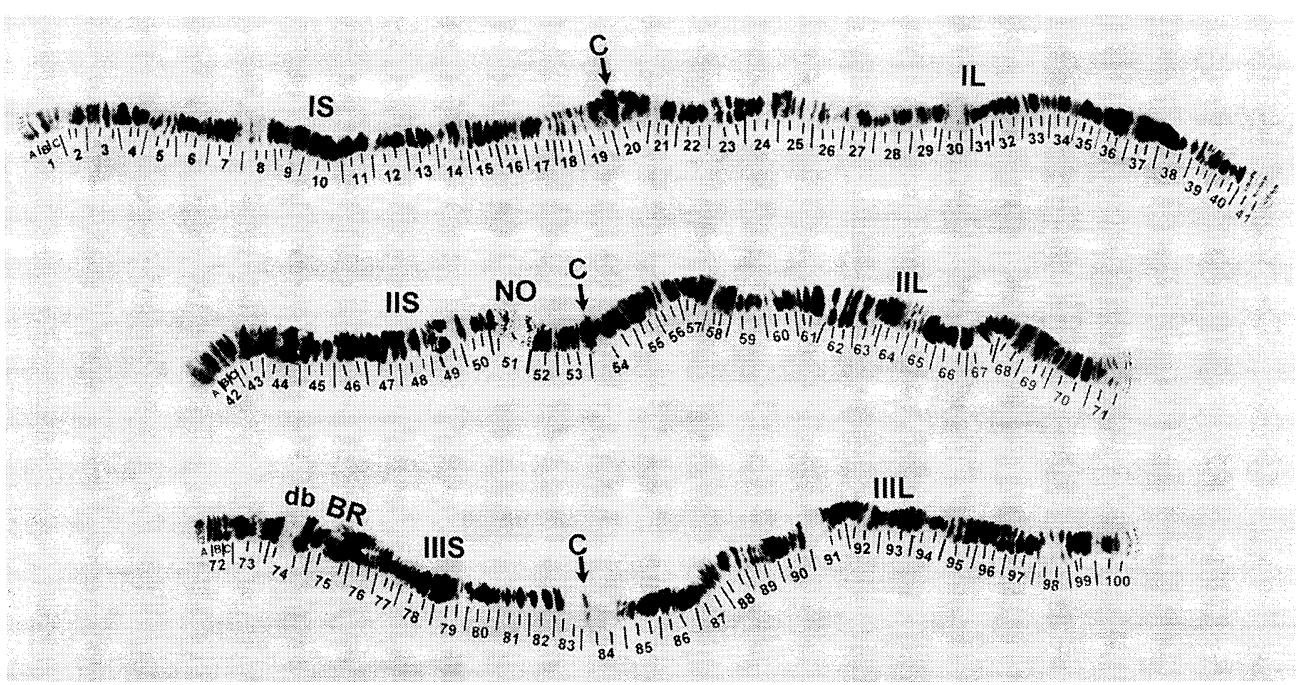

Fig. 7. Standard photographic map of salivary gland polytene chromosomes of Simulium (Simulium) rufibasis. Symbols and designations as in Fig. 2.

\section{S. (S.) rufibasis}

Chromosome I is metacentric with approximately $41 \%$ TCL. The short arm expands from sections 1-19 while the long arm occupies the remaining sections 20-41 (Fig. 7). A group of 5 heavy bands in sections $14 \mathrm{~A}-16 \mathrm{~A}$ is a good landmark for the short arm.

Chromosome II is submetacentric with $30 \%$ TCL. The short arm is characterized by the presence of a nucleolar organizer. A prominent three dark bands in sections $62 \mathrm{~A}-\mathrm{C}$ is useful for cytological landmark for the long arm.

Chromosome III is metacentric with $29 \%$ TCL, divided into 29 sections (72-100). The short arm is recognized by the presence of double bubble and the Ring of Balbiani. A series of dark bands in sections 95B-97C is a good character for the long arm.

No inversions have been found in any chromosome arms. 


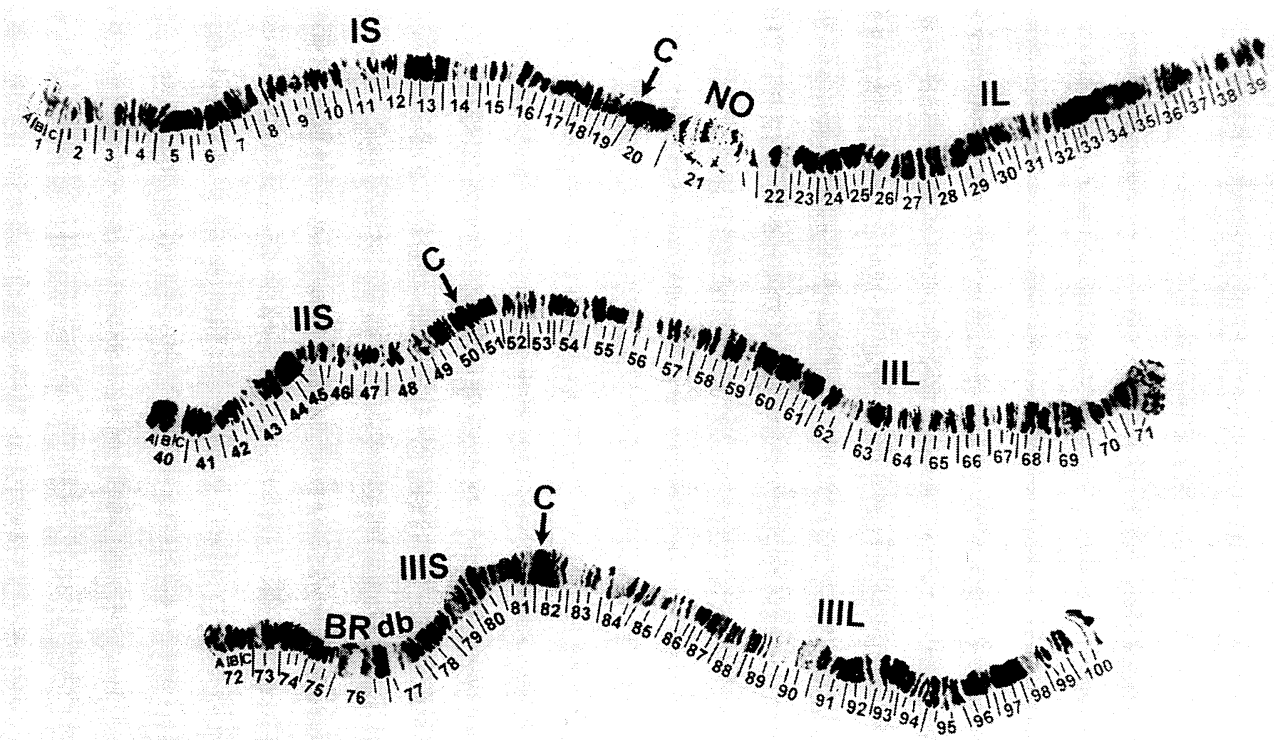

Fig. 8. Standard photographic map of salivary gland polytene chromosomes of Simulium (Montisimulium) sp. G. Symbols and designations as in Fig. 2.

\section{S. (M.) sp. $G$}

Chromosome I is metacentric, the short arm and the long arm being of almost equal length. It is about 39\% TCL, divided into 19 sections (1-19) for the short arm and 20 sections (20-39) for the long arm (Fig. 8). The short arm have two prominent series of dark bands in sections $12 \mathrm{C}$ and $13 \mathrm{~B}$ respectively. The long arm is differentiated by the presence of a prominent nucleolar organizer.

Chromosome II is submetacentric and contains 32 sections (40-71). The short arm is recognized by the presence of a series of four dark bands at a blunt tip. On the other hand, a large flared tip in sections $71 \mathrm{BC}$ is a useful cytological landmark for the long arm.

Chromosome III is also submetacentric and shortest of the complement. It is divided into 29 sections (72-100). The presence of the Ring of Balbiani in section 76A and the double bubble in section 77B can distinguish the short arm from the long arm. As in other species except for $S$. (S.) fenestratum, the 3 dark bands adjacent to the centromere of the short arm serve as a useful landmark. The long arm have 2 groups of dark bands in sections 93A-94A and sections 97A-98A respectively. Moreover, a flared tip is also a good character for the long arm.

No inversions have been detected in all three chromosomes.

\section{Homology of the banding sequences}

The banding patterns of 3 Simulium species, $S$. (S.) nakhonense, $S$. (S.) rufibasis and $S$. (M.) sp. G compared with the standard sequences of $S$. $(N$.) caudisclerum showed different sequences except for some banding sequences in the short arm of chromosome III.

In the short arm of chromosome III, the banding sections from double bubble to the Ring of Balbiani of $S$. $(N$.) caudisclerum (sections 76C-77C), and $S$. (S.) rufibasis (sections 74B-75B) are homologous (Fig. 9a, b). These banding sections are also homologous with the inverted banding sections from the Ring of Balbiani to double bubble of $S$. (M.) sp. G (sections 76A-77B) and $S$. (S.) nakhonense (sections 75B-76C) (Fig. 9c, d). In addition, the 3 dark bands adjacent to the centromeres of $S$. (S.) rufibasis (sections 82A-83A), $S$. (M.) sp. G (sections $81 \mathrm{~A}-\mathrm{C}$ ) and $S$. (S.) nakhonense (sections $82 \mathrm{C}-83 \mathrm{~B}$ ) compared with the standard sequence of $S$. (N.) caudisclerum (sections $81 \mathrm{C}-82 \mathrm{~B}$ ) were also found to be homologous (Fig. 9a-d). 


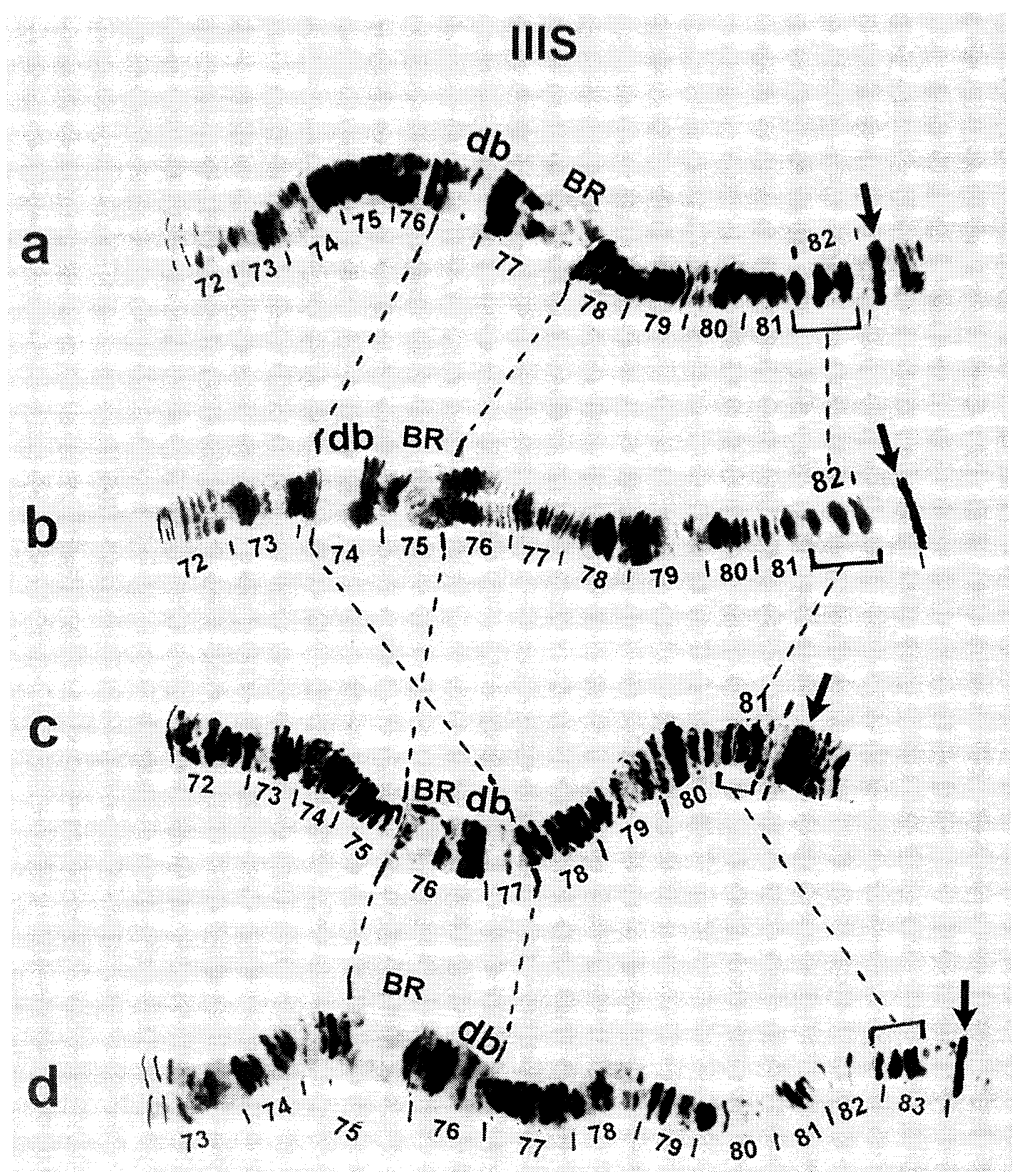

Fig. 9. Homologies of salivary gland polytene chromosome arm IIIS of Simulium (N.) caudisclerum (a), S. (S.) rufibasis (b), S. (M.) sp. G (c) and $S$. (S.) nakhonense (d). Centromeres are indicated by arrows. Broken lines indicate homologous bands.

\section{Discussion}

The 5 species of Simulium cytologically studied here have 3 pairs of polytene salivary gland chromosomes $(2 n=6)$ as already reported in most other species of Simulium (Bedo 1977, Rothfels 1979, Boakye 1993, Hadi et al. 1996a, b, c). In all 5 species, the chromosomal landmarks, such as the nucleolar organizer, the Ring of Balbiani and double bubble are located in the same or different chromosomes. The prominent nucleolar organizer is located in chromosome I of all species except $S$. (S.) rufibasis, located in chromosome II, which is distinct character of this species. The Ring of Balbiani and double bubble of $S$. (S.) fenestratum are near the tip of the short arm of chromosome II as found in S. yaeyamaense (Hadi et al. 1995), S. bidentatum, S. aokii and S. arakawae (Hadi et al. 1996a) and S. sundaicum (Hadi et al. 1996c). On the other hand, in $S$. (N.) caudisclerum, $S$. (S.) rufibasis, $S$. (S.) nakhonense and $S$. (M.) sp. G, those landmarks are in the short arm of chromosome III.

The centromeric regions of polytene chromosomes of $S$. (N.) caudisclerum, $S$. (S.) fenestratum and $S$. (M.) sp. G, show the existence of dark band as found in S. yaeyamaense (Hadi et al. 1995). This is different from those of $S$. (S.) nakhonense and $S$. (S.) rufibasis, in which centromeric region is distinctly expanded and easily recognized as reported in S. bidentatum, S. aokii and $S$. 
arakawae (Hadi et al. 1996a), S. eximium (Hadi et al. 1996b) and S. sundaicum (Hadi et al. 1996c). The difference of locations of certain landmarks, as well as that of centromeric banding pattern, seems to be specific to the species but not to the subgenus. However, more specimens of other related species in the same subgenera from various localities are needed for further confirmation.

In some larvae of $S$. ( $S$.) nakhonense, two types of B chromosomes occurred independently as a small metacentrics, as reported in S. bidentatum and $S$. aokii (Hadi et al. 1996a). B chromosomes have been also reported in other blackfly species such as $S$. vernum group (Brockhouse et al. 1989) and $S$. ochraceum (Hirai et al. 1994). The role and effect of B chromosome in Simuliidae are still obscure and remain to be studied. Moreover, polytene chromosomes of some larvae of $S$. (S.) nakhonense were ectopically paired forming a loose pseudochromocenter. In S. melatum, larval salivary gland chromosomes are also held together by a loose pseudochromocenter (Bedo 1975), while adult malpighian chromosomes are held together by a very tight chromocenter preventing separation of individual centromere (Bedo 1977). Pseudochromocenter were also found in some species of Prosimulium (Basrur 1962, Ottonen 1966, Rothfels and Freeman 1977), S. costatum (Brockhouse et al. 1989) and S. squamosum (Boakye 1993).

The arrange of the sections between the Ring of Balbiani and double bubble in the short arm of chromosome III of $S$. (N.) caudisclerum and $S$. (S.) rufibasis are similar, in which the Ring of Balbiani is close to the centromere. In $S$. (S.) nakhonense and $S$. (M.) sp. G, these sections seem to be derived from those of $S$. ( $N$.) caudisclerum which belongs to subgenus Nevermannia, the most primitive subgenus, but they are inverted positions. This leads to change the positions of double bubbles being close to the centromeres.

Species which share gene arrangements are more related than those that do not. Those species may have originated from a common ancestor. The 3 dark bands adjacent to the centromeres of the short arm of chromosome III in 4 Simulium species, $S$. (N.) caudisclerum, $S$. (S.) rufibasis, $S$. (S.) nakhonense and $S$. (M.) sp. G may derived from common ancestor and subsequent fixation in each species of the same and different subgenera.

\section{Acknowledgements}

We thank Professor Hiroyuki Takaoka for his kind identification of larval and pupal specimens and also for his constructive criticism of the manuscript. This work was supported by the TRF/BIOTEC Special Programme for Biodiversity Research and Training Grant BRT 139007.

\section{References}

Basrur, P. K. 1962. The salivary gland chromosomes of seven species of Prosimulium (Dipters: Simuliidae) from Alaska and British Columbia. Can. J. Zool. 40: 1019-1033.

Bedo, D. G. 1975. C-banding in polytene chromosomes of Simulium ornatipes and S. melatum (Diptera: Simuliidae). Chromosoma (Berl.) 51: 291-300.

- 1977. Cytogenetics and evolution of Simulium ornatipes Skuse (Diptera: Simuliidae). I. Sibling speciation. Chromosoma 64: 37-65.

— 1979. Cytogenetics and evolution of Simulium ornatipes Skuse (Diptera: Simuliidae). II. Temporal variation in chromosome polymorphisms and homosequential sibling species. Evolution 33: 482-507.

Boakye, D. A. 1993. A pictoral guide to the chromosomal identification of members of the Simulium damnosum Theobald complex in West Africa with particular reference to the Onchocerciasis Control Programme Area. Trop. Med. Parasitol. 44: 223-244.

Brockhouse, C., Bass, J. A. B. and Straus, N. A. 1989. Chromocentre polymorphism in polytene chromosomes of Simulium costatum (Diptera: Simuliidae). Genome 32: 510-515.

Conn, J., Rothfels, K. H., Procunier, W. S. and Hirai, H. 1989. The Simulium metallicum species complex (Diptera: Simuliidae) in Latin America: a cytological study. Can. J. Zool. 67: 1217-1245.

Crosskey, R. W. 1960. A taxonomic study of the larvae of West African Simuliidae (Diptera: Simuliidae) with comments on the morphology of the larval black-fly head. Bull. Br. Mus. Nat. Hist. (Ent.) 10: 1-74. 
— 1981. Simuliid Taxonomy. In: Laird, M. (ed.), Blackflies: The Future for Biological Methods in Integrated Control. Academic Press, London. pp. 3-18.

- 1993. Blackflies (Simuliidae). In: Lane R. P. and R. W. Crosskey (ed.), Medical Insects and Arachnids. Chapman and Hall, New York. pp. 241-287.

Datta, M. 1974a. Some black flies (Diptera: Simuliidae) of the subgenus Simulium latreille (s. str.) from the Darjeeling area, India. Oriental Insects 8: 15-27.

— 1974b. New species of black flies (Diptera: Simuliidae) from the Darjeeling area, India. Oriental Insects 8: 457-468.

Davies, L. 1968. A key to the British species of Simuliidae (Diptera) in the larval, pupal and adult stages. Freshwater Biological Association, Scientific Publication No. 24: 3-85.

Dunbar, R. W. and Vajime, C. G. 1981. Cytotaxonomy of the Simulium damnosum Complex. In: Laird, M. (ed.), Blackflies: The Future for Biological Methods in Integrated Control. pp. 31-43.

Hadi, U. K., Takaoka, H. and Aoki, C. 1996a. Larval polytene chromosomes of three Japanese blackfly species (Diptera: Simuliidae). Med. Entomol. Zool. 47: 47-54.

- - - and Kondo, K. and Hirai, H. 1996b. Larval salivary gland chromosomes of Simulium (Simulium) eximium (Diptera; Simuliidae) from Java and Sumatra, Indonesia, with implication of sibling speciation. Med. Entomol. Zool. 47: 139-144.

—, - - - and - 1996c. Larval salivary gland chromosomes of the blackfly, Simulium (Gomphostilbia) sundaicum (Diptera: Simuliidae) from Java, Indonesia. Med. Entomol. Zool. 47: 55-61.

Hirai, H., Procumier, W. S., Ochoa, J. O. and Uemoto, K. 1994. A cytogenetic analysis of the Simulium ochraceum species complex (Diptera: Simuliidae) in Central America. Genome 37: 36-53.

Kuvangkadilok, C., Phayuhasena, S. and Baimai, V. 1999. Population cytogenetic studies on Simulium feuerborni Edwards (Diptera: Simuliidae) from northern Thailand. Genome (in press).

Mavragani-Tsipidou, P., Zambetaki, A., Kleanthous, K., Pangou, E. and Scouras, Z. G. 1994. Cytotaxonomic differentiation of the Afrotropical Drosophila montium subgroup: D. diplacantha and D. seguyi. The major role of reverse tandem duplications. Genome 37: 935-944.

Ottonen, P. O. 1966. The salivary gland chromosomes of six species in the IIIS-1 group of Prosimulium Roub. (Diptera: Simuliidae). Can. J. Zool. 44: 677-701.

Porter, D. L. and Martin, J. 1977. The cytology of Polypedilum nubifer (Diptera: Chironomidae). Caryologia 30: 41-62.

Procunier, W. S. and Muro, A. I. 1993. Cytotaxonomy of the Simulium damnosum complex from central and northeastern Tanzania. Genome 36: 112-130.

— and Smith, J. J. 1993. Localization of ribosomal DNA in Rhagoletis pomonella (Diptera: Tephritidae) by in situ hybridization. Insect Mol. Biol. 2: 163-174.

Rothfels, K. H. 1979. Cytotaxonomy of black flies (Simuliidae). Ann. Rev. Entomol. 24: 507-539.

— and Freeman, M. 1977. The salivary gland chromosomes of seven species of Prosimulium (Diptera: Simuliidae) in the mixtum (IIIL-1) group. Can. J. Zool. 55: 482-507.

Sukowati, S. and Baimai, V. 1996. A standard cytogenetic map for Anopheles sundaicus (Diptera: Culicidae) and evidence for chromosomal differentiation in populations from Thailand and Indonesia. Genome 39: 165-173.

Takaoka, H. 1977. Studies on black flies of the Nansei Island, Japan (Simuliidae: Diptera). III. In six species of the subgenus Simulium Latreille. Jpn. J. Sanit. Zool. 28: 193-217.

— 1979. The black flies of Taiwan (Diptera: Simuliidae). Pacific Insects 20: $365-403$.

— and Suzuki, H. 1984. The blackflies (Diptera: Simuliidae) from Thailand. Jpn. J. Sanit. Zool. 35: 7-45.

— and Davies, D. M. 1995. The Black Fles (Diptera: Simuliidae) of West Malaysia. Fukuoka: Kyushu University Press. 175 pp.

— and Saito, K. 1996. A new species and new records of black flies (Diptera: Simuliidae) from Thailand. Jpn. J. Trop. Med. Hyg. 24: 163-169.

— and Adler, P. H. 1997. A new subgenus, Simulium (Daviesellum), and a new species, S. (D.) caurtneyi, (Diptera: Simuliidae) from Thailand and Peninsular Malaysia. Jpn. J. Trop. Med. Hyg. 28: 17-27.

Vajime, C. G. and Dunbar, R. W. 1975. Chromosomal identification of eight species of the subgenus Edwardsellum near and including Simulium (Edwardsellum) damnosum Theobald (Diptera: Simuliidae). Tropenmed. Parasit. 26: 111-138.

Wülker, W., Sublette, J. E., Morath, E. and Martin, J. 1989. Chironomus anonymus Williston in North America-closely related species. Studies on Neotropical Fauna and Environment 24: 121-136. 